\title{
Time-Hopping Code Characterization for Multi-User Interference Mitigation in Ultra Wide Band Impulse Radio
}

\author{
Anne-Laure Deleuze ${ }^{1,2}$, Christophe J. Le Martret ${ }^{1}$ and Philippe Ciblat ${ }^{2}$ \\ ${ }^{1}$ THALES Land and Joint Systems, Colombes, France \\ Email: anne-laure.deleuze@ fr.thalesgroup.com, christophe.le_martret@ fr.thalesgroup.com \\ ${ }^{2}$ École Nationale Supérieure des Télécommunications, Paris, France \\ Email: deleuze@enst.fr, philippe.ciblat@enst.fr
}

\begin{abstract}
We consider an ultra wide band impulse radio multiple access scheme based on time-hopping code under a realistic multipath channel model. As a novelty, we provide a closed-form expression for the variance of the multi-user interference at the output of the rake receiver of the user of interest. That enables us to characterize the optimal time-hopping codes minimizing the multi-user interference variance. Finally we observe through simulations that minimizing the multi-user interference variance translates into better performance by significantly decreasing the error probability.
\end{abstract}

\section{INTRODUCTION}

Ultra Wide Band impulse radio (UWB-IR) based communication system has known a growing interest for the last decade. Associated with a multiple access technique such as the time-hopping, this modulation scheme is especially a good candidate for short range wireless communication based network in dense multipath environments.

In this paper, we focus on asynchronous Time-Hopping Codes (THC) impulse radio multiple access demodulated by a rake receiver as described for instance in [1]. In synchronous (or quasi-synchronous) links, the multi-user interference (MUI) may be canceled by the use of orthogonal codes (see e.g., [2] for application to UWB communication systems). Conversely, in asynchronous transmissions, the MUI cannot be nulled and give rise to bit error rate (BER) floor which limits the system performance. Different works have tackled the characterization of the MUI in order to predict the performance of the system. Many of them have modeled the MUI as a random Gaussian process either in free-space propagation (see e.g., [1]), or in multipath channels [3]. Due to this assumption, the resulting MUI does not depend on the code realization and thus no code optimization is possible. Based upon a general channel model, and assuming that the codes as deterministic, we show that the variance of the MUI can be computed in a closed-form expression with respect to the THC sequence. Thus, this gives a way to mitigate the effect of the MUI by selecting the codes carefully.

This paper is organized as follows: in Section II, we introduce the time-hopping UWB-IR based system. In Section III, the closed-form expression of the MUI variance at the output of the rake receiver is provided. In Section IV, we discuss about the THC selection. In Section V, we present simulations results particularly the error probability for different THCs selections. Finally, some conclusions are given in Section VI.

\section{SySTEM MOdEL}

\section{A. Transmitted and received signals}

We consider a Pulse Amplitude Modulation ${ }^{1}$ (PAM) UWBIR communication system which employs the time-hopping scheme as code-division multiple-access technique [4]. We consider that $N_{u}$ users are active in the network. These users transmit information asynchronously through different propagation channels.

The transmitted signal from user $n$ can be written as follows:

$$
s_{n}(t)=\sum_{i=-\infty}^{+\infty} d_{n}\left(\left\lfloor i / N_{f}\right\rfloor\right) w\left(t-i T_{f}-\tilde{c}_{n}(i) T_{c}-\theta_{n}\right),
$$

where

- $N_{c}$ is the number of chips of duration $T_{c}$,

- $N_{f}$ is the number of frames of duration $T_{f}=N_{c} T_{c}$,

- $w(t)$ is the impulse of duration $T_{w} \ll T_{c}$,

- $\lfloor x\rfloor$ is the integer-floor of $x$,

- $d_{n}(i) \in\{-1,1\}$ are the transmitted symbols, assumed to be independent and identically distributed,

- $\theta_{n}$ represents the time asynchronism, modeled as random variable uniformly distributed over a symbol period $\left[0, N_{f} T_{f}\right)$,

- $\left\{\tilde{c}_{n}(i)\right\} \in\left\{0, \cdots, N_{c}-1\right\}$ are the THC assumed to be periodic of period $N_{f}$.

In order to derive the MUI variance, it is convenient to use the so-called developed time-hopping code (DTHC) sequence, denoted $\left\{c_{n}(j)\right\}_{j=0}^{N_{c} N_{f}-1}$, and firstly introduced in

\footnotetext{
${ }^{1}$ All the results presented here can be extended to the PPM signal in the similar way [5].
} 
[2]. The DTHC sequence is deduced from the THC sequence $\left\{\tilde{c}_{n}(i)\right\}_{i=0}^{N_{f}-1}$ as follows:

$$
c_{n}(j)= \begin{cases}1 & \text { if } j=\tilde{c}_{n}(i)+i N_{c}, 0 \leq i \leq N_{f}-1, \\ 0 & \text { otherwise. }\end{cases}
$$

Consequently, (1) takes the following form

$$
s_{n}(t)=\sum_{i=-\infty}^{+\infty} d_{n}(i) \sum_{j=0}^{N_{c} N_{f}-1} c_{n}(j) w\left(t-i N_{f} T_{f}-j T_{c}-\theta_{n}\right)
$$

At the receiver (which is assumed to be different from all the active users), the signal is corrupted by a multipath channel. Thus the received signal can be expressed as follows:

$$
r(t)=\sum_{n=1}^{N_{u}} \sum_{k=1}^{N_{p}} A_{n}^{k} s_{n}\left(t-\tau_{n}^{k}\right)+n(t),
$$

where

- $A_{n}^{k}$ and $\tau_{n}^{k}$ are the amplitude and the delay of the $k^{\text {th }}$ path respectively between the user $n$ and the receiver,

- $N_{p}$ is the number of paths,

- $N_{u}$ is the number of active users,

- $n(t)$ is an additive zero-mean white Gaussian noise.

For the sake of simplicity, we have assumed that the number of paths is the same for all the users but generalization to a different number of paths per user is straightforward. In the sequel, without loss of generality, we also consider that the delays have been sorted such as $\forall n, k, \tau_{n}^{k}<\tau_{n}^{k+1}$ holds.

\section{B. Channel statistics}

We will consider a statistical model for the different multipath propagation channels that enables us to encompass most of the models proposed so far for the UWB schemes.

For modeling a multipath channel, it is usual to assume that the amplitude $A_{n}^{k}$ depends on the corresponding delay $\tau_{n}^{k}$. We propose to model this dependency by setting $A_{n}^{k}=a_{n}^{k} \cdot f\left(\tau_{n}^{k}\right)$ where $a_{n}^{k}$ is a random variable that accounts for the statistics of the amplitude (independent of $\tau_{n}^{k}$ ) and $f(\cdot)$ is a function that accounts for the decaying of the amplitude with respect to the delay. The random variables $a_{n}^{k}$ are assumed to be independent and zero mean with variance $\sigma_{a}^{2}:=\mathbb{E}_{a}\left[\left(a_{n}^{k}\right)^{2}\right]$ and thus check $\mathbb{E}_{a}\left[a_{n_{1}}^{k_{1}} a_{n_{2}}^{k_{2}}\right]=\mathbb{E}_{a}\left[a_{n_{1}}^{k_{1}}\right] \mathbb{E}_{a}\left[a_{n_{2}}^{k_{2}}\right]=0$, except for the case $k_{1}=k_{2}$ and $n_{1}=n_{2}$. In the following we put $I_{n}^{k}:=\mathbb{E}_{a}\left[\left(A_{n}^{k}\right)^{2}\right]=\sigma_{a}^{2} \cdot f^{2}\left(\tau_{n}^{k}\right)$. The random variables $\tau_{n}^{k}$ are assumed to be independent between users but are usually correlated for a given user. Most of the channel models proposed for the UWB can be cast into this general model, and more particularly, the recent modified Saleh-Valenzuela model, which has been selected in the TG3a for the IEEE 802.15.3a standard [6]. In this model, when only one cluster is considered (as it will be in the simulation section of this paper), the delay $\tau_{n}^{k}$ follows a Poisson distribution with parameter $\lambda$. Furthermore, the amplitude $a_{n}^{k}=p_{n}^{k} \cdot \beta_{n}^{k}$, where $p_{n}^{k}$ is equiprobable \pm 1 and $\beta_{n}^{k}$ is a log-normal random variable. Notice that, in the original Saleh-Valenzuela model [7], the amplitudes $a_{k}^{n}$ are real zero mean Gaussian random variables.

The function $f(\cdot)$ is chosen as follows: $f\left(\tau_{n}^{k}\right)=e^{-\tau_{n}^{k} / \gamma}$ where $\gamma$ is the path power-decay time.

\section{Rake receiver}

We assume here a generic rake receiver that may select any subset $\mathscr{L}$ of $L_{r}$ paths (with $L_{r} \leq N_{p}$ ). Let assume that the receiver demodulates symbols of one of the active users, let say user 1 . Then, the rake output can be written as (assuming $\left.\theta_{1}=0\right)$

$$
z=\sum_{\ell \in \mathscr{L}} A_{1}^{\ell} \int_{0}^{N_{f} T_{f}} r\left(t+\tau_{1}^{\ell}\right) \times \underbrace{\sum_{j=0}^{N_{c} N_{f}-1} c_{1}(j) w\left(t-j T_{c}\right)}_{v_{1}(t)} d t
$$

where $v_{1}(t)$ is the receiver template for user 1 . By putting (4) into (5) we obtain :

$$
z=\sum_{\ell \in \mathscr{L}} A_{1}^{\ell} \sum_{n=1}^{N_{u}} \sum_{k=1}^{N_{p}} A_{n}^{k} y_{k, \ell, n}\left(\theta_{n}\right)+\eta,
$$

where $\eta:=\sum_{\ell \in \mathscr{L}} A_{1}^{\ell} \int_{0}^{N_{f} T_{f}} n\left(t+\tau_{1}^{\ell}\right) v_{1}(t) d t$ is the filtered noise, and

$$
\begin{aligned}
y_{k, \ell, n}\left(\theta_{n}\right):= & \sum_{i=-\infty}^{+\infty} d_{n}(i) \sum_{j=0}^{N_{c} N_{f}-1} c_{n}(j) \int_{0}^{N_{f} T_{f}} v_{1}(t) . \\
& w\left(t-i N_{f} T_{f}-j T_{c}-\theta_{n}-\Delta \tau_{k, \ell, n}\right) d t
\end{aligned}
$$

with $\Delta \tau_{k, \ell, n}:=\tau_{n}^{k}-\tau_{1}^{\ell}$. Expression (6) can be separated into four terms

$$
z:=z_{1}+z_{2}+z_{3}+\eta
$$

with

$$
\begin{aligned}
z_{1} & =\sum_{\ell \in \mathscr{L}}\left(A_{1}^{\ell}\right)^{2} y_{\ell, \ell, 1}(0), \\
z_{2} & =\sum_{\ell \in \mathscr{L}} A_{1}^{\ell} \sum_{k \neq \ell=1}^{N_{p}} A_{1}^{k} y_{k, \ell, 1}(0), \\
z_{3} & =\sum_{\ell \in \mathscr{L}} A_{1}^{\ell} \sum_{n=2}^{N_{u}} \sum_{k=1}^{N_{p}} A_{n}^{k} y_{k, \ell, n}\left(\theta_{n}\right),
\end{aligned}
$$

which can be interpreted as follows:

- $z_{1}$ is the energy collected from the user of interest,

- $z_{2}$ is the inter-symbol/inter-frame interference (ISI/IFI) for the user of interest,

- $z_{3}$ is the multiuser interference.

The energy $z_{1}$ and the filtered noise $\eta$ do not depend on the code selection whereas $z_{2}$ and $z_{3}$ do. In practical systems the ISI/IFI can be chosen arbitrarily small regardless of the codes by inserting a guard time, larger than the maximum delay spread of the channel, placed at the end of each frame (see e.g., [4]). In contrast, the MUI is inherent of the Time-Hopping UWB-IR transmission scheme and can be mitigated only by designing the codes properly. Therefore, as mentioned in the introduction, the rest of the paper will be dedicated to the research of optimal codes that minimize the variance of the MUI.

As $z_{3}$ depends on random variables $a_{n}^{k}, d_{n}, \theta_{n}$ and $\tau_{n}^{k}$, we would like to know that the variance of MUI averaged over 
all these nuisance parameters. Therefore, the objective of the paper is to derive in closed-form expression the variance of the MUI, $\sigma_{3}^{2}:=\mathbb{E}_{a, d, \theta, \tau}\left[z_{3}^{2}\right]$.

\section{Closed-FORM EXPRESSION FOR THE MUI VARIANCE}

The purpose of this section is to evaluate the MUI variance $\sigma_{3}^{2}$ with respect to the DTHC. In order to derive this variance, we firstly need the following lemma.

Lemma 1 Let $\theta_{n}+\Delta \tau_{k, \ell, n}=Q_{n}^{k, \ell} N_{f} T_{f}+q_{n}^{k, \ell} T_{c}+\varepsilon_{n}^{k, \ell}$, where

- $Q_{n}^{k, \ell}=\left\lfloor\left(\theta_{n}+\Delta \tau_{k, \ell, n}\right) / N_{f} T_{f}\right\rfloor$,

- $q_{n}^{k, \ell}=\left\lfloor\left(\theta_{n}+\Delta \tau_{k, \ell, n}-Q_{n}^{k, \ell} N_{f} T_{f}\right) / T_{c}\right\rfloor$,

- $\varepsilon_{n}^{k, \ell} \in\left[0, T_{c}\right)$, the remainder.

Let $r_{w w}(s):=\int_{-\infty}^{+\infty} w(t) w(t-s) d t$. As the time support of $r_{w w}$ is much less than $T_{c}$, we get:

$$
\begin{aligned}
y_{k, \ell, n}\left(\theta_{n}\right)= & d_{n}\left(-Q_{n}^{k, \ell}\right)\left[\mathscr{C}_{1, n}^{+}\left(q_{n}^{k, \ell}\right) r_{w w}\left(\varepsilon_{n}^{k, \ell}\right)+\right. \\
& \left.\mathscr{C}_{1, n}^{+}\left(q_{n}^{k, \ell}+1\right) r_{w w}\left(\varepsilon_{n}^{k, \ell}-T_{c}\right)\right]+ \\
& d_{n}\left(-Q_{n}^{k, \ell}-1\right)\left[\mathscr{C}_{1, n}^{-}\left(q_{n}^{k, \ell}\right) r_{w w}\left(\varepsilon_{n}^{k, \ell}\right)+\right. \\
& \left.\mathscr{C}_{1, n}^{-}\left(q_{n}^{k, \ell}+1\right) r_{w w}\left(\varepsilon_{n}^{k, \ell}-T_{c}\right)\right]
\end{aligned}
$$

with

$$
\begin{aligned}
\mathscr{C}_{m, n}^{-}(q) & =\sum_{k=0}^{q-1} c_{m}(k) c_{n}(k-q), \\
\mathscr{C}_{m, n}^{+}(q) & =\sum_{k=q}^{N_{c} N_{f}-1} c_{m}(k) c_{n}(k-q) .
\end{aligned}
$$

Proof: Using the definition of $v_{1}(t)$ given in (5) leads to

$$
\begin{array}{r}
y_{k, \ell, n}\left(\theta_{n}\right)=\sum_{i=-\infty}^{+\infty} d_{n}(i) \sum_{j=0}^{N_{c} N_{f}-1} \sum_{j_{1}=0}^{N_{c} N_{f}-1} c_{n}(j) c_{1}\left(j_{1}\right) \\
r_{w w}\left(\left(Q_{n}^{k, \ell}+i\right) N_{f} T_{f}+\left(q_{n}^{k, \ell}+j-j_{1}\right) T_{c}+\varepsilon_{n}^{k, \ell}\right) .
\end{array}
$$

As the time support of $r_{w w}$, denoted by $T_{r_{w w}}$ is less than $T_{c}$, the terms contributing to the previous equation are non-null if and only if

$-T_{r_{w w}} \leq\left(Q_{n}^{k, \ell}+i\right) N_{f} T_{f}+\left(q_{n}^{k, \ell}+j-j_{1}\right) T_{c}+\varepsilon_{n}^{k, \ell} \leq T_{r_{w w}}$.

As $\Delta \tau_{k, \ell, n} \geq 0$ (the case $\Delta \tau_{k, \ell, n} \leq 0$ can be achieved in a similar way) and, $-N_{c} N_{f}+1 \leq q_{n}^{k, \ell}+j-j_{1} \leq 2 N_{c} N_{f}-2$, the non-null terms in $y_{k, \ell, n}\left(\theta_{n}\right)$ are obtained for $Q_{n}^{k, \ell}+i=0$ or -1 . We derive the term for $Q_{n}^{k, \ell}+i=0$. Thus it implies $j-j_{1}+q_{n}^{k, \ell}$ is equal to -1 or 0 . The term in $y_{k, \ell, n}\left(\theta_{n}\right)$ for $Q_{n}^{k, \ell}+i=0$ takes thus the following form:

$d_{n}\left(-Q_{n}^{k, \ell}\right)\left[\mathscr{C}_{1, n}^{+}\left(q_{n}^{k, \ell}\right) r_{w w}\left(\varepsilon_{n}^{k, \ell}\right)+\mathscr{C}_{1, n}^{+}\left(q_{n}^{k, \ell}+1\right) r_{w w}\left(\varepsilon_{n}^{k, \ell}-T_{c}\right)\right]$.

The derivation for the term associated with the condition $Q_{n}^{k, \ell}+i=-1$ can be done in a similar way and provides the second part of (12).
Quantities $\mathscr{C}_{m, n}^{-}(q)$ and $\mathscr{C}_{m, n}^{+}(q)$ can be viewed as a partial cyclic cross-correlation between user $m$ and user $n$.

Since the random variables involved in $z_{3}$ are assumed independent, we can compute the expectation independently one after another. For convenience purpose, we present the computation taking the expectations in the following order: $a_{n}^{k}, d_{n}$, and then $\theta_{n}, \tau_{n}^{k}$. From (11) and (12) and after straightforward derivations, the expectation of $z_{3}^{2}$ over the amplitude and the symbol is given by:

$$
\begin{array}{r}
\mathbb{E}_{a, d}\left[z_{3}^{2}\right]=\sum_{n, k, \ell} I_{n}^{k} I_{1}^{\ell}\left[\left(\left(\mathscr{C}_{1, n}^{+}\left(q_{n}^{k, \ell}\right)\right)^{2}+\left(\mathscr{C}_{1, n}^{-}\left(q_{n}^{k, \ell}\right)\right)^{2}\right) r_{w w}^{2}\left(\varepsilon_{n}^{k, \ell}\right)+\right. \\
\left.\left(\left(\mathscr{C}_{1, n}^{+}\left(q_{n}^{k, \ell}+1\right)\right)^{2}+\left(\mathscr{C}_{1, n}^{-}\left(q_{n}^{k, \ell}+1\right)\right)^{2}\right) r_{w w}^{2}\left(\varepsilon_{n}^{k, \ell}-T_{c}\right)\right] .
\end{array}
$$

For a fixed $\Delta \tau_{k, \ell, n}$ value, when $\theta_{n}$ ranges from 0 to $N_{f} T_{f}$, $\varepsilon_{n}^{k, \ell, n}$ ranges from 0 to $T_{c}$, periodically $\left(N_{c} N_{f}-1\right)$ times. Thus the expectation over $\theta_{n}$ yields:

$$
\begin{aligned}
\mathbb{E}_{a, d, \theta}\left[z_{3}^{2}\right]= & \frac{1}{N_{f} T_{f}} \sum_{n, k, \ell, q} I_{n}^{k} I_{1}^{\ell}\left[\left(\left(\mathscr{C}_{1, n}^{+}(q)\right)^{2}+\left(\mathscr{C}_{1, n}^{-}(q)\right)^{2}\right) \gamma_{1}+\right. \\
& \left.\left(\left(\mathscr{C}_{1, n}^{+}(q+1)\right)^{2}+\left(\mathscr{C}_{1, n}^{-}(q+1)\right)^{2}\right) \gamma_{2}\right],
\end{aligned}
$$

with

$$
\gamma_{1}:=\int_{0}^{T_{c}} r_{w w}^{2}(\varepsilon) d \varepsilon \text { and } \gamma_{2}:=\int_{0}^{T_{c}} r_{w w}^{2}\left(\varepsilon-T_{c}\right) d \varepsilon
$$

Using the codes periodicity we have:

$\sum_{q}\left(\mathscr{C}_{1, n}^{+}(q)\right)^{2}+\left(\mathscr{C}_{1, n}^{-}(q)\right)^{2}=\sum_{q}\left(\mathscr{C}_{1, n}^{+}(q+1)\right)^{2}+\left(\mathscr{C}_{1, n}^{-}(q+1)\right)^{2}$, and putting

$$
\gamma_{w w}:=\int_{-T_{c}}^{T_{c}} r_{w w}^{2}(\varepsilon) d \varepsilon
$$

we get

$\mathbb{E}_{a, d, \theta}\left[z_{3}^{2}\right]=\frac{1}{N_{f} T_{f}} \sum_{n, k, \ell, q} I_{n}^{k} I_{1}^{\ell}\left(\left(\mathscr{C}_{1, n}^{+}(q)\right)^{2}+\left(\mathscr{C}_{1, n}^{-}(q)\right)^{2}\right) \gamma_{w w}$.

In the previous equation, only the terms $I_{n}^{k}$ still depend on the delays. Consequently the expectation over the delays leads to:

$$
\sigma_{3}^{2}=\frac{\psi \gamma_{w w}}{N_{f} T_{f}} \kappa_{1}
$$

where

$$
\begin{aligned}
\kappa_{m} & :=\sum_{\substack{n=1 \\
n \neq m \\
n \neq m}}^{N_{u}} \kappa_{m, n}, \\
\kappa_{m, n} & :=\sum_{q=0}^{N_{f}-1}\left(\mathscr{C}_{m, n}^{+}(q)\right)^{2}+\left(\mathscr{C}_{m, n}^{-}(q)\right)^{2}, \\
\psi & :=\sum_{\ell \in \mathscr{L}} \mathbb{E}_{\tau}\left[I_{1}^{\ell}\right] \sum_{k=1}^{N_{p}} \mathbb{E}_{\tau}\left[I_{n}^{k}\right] .
\end{aligned}
$$

The computation of the variance of the MUI has been tackled by other authors. The earliest work can be found in [1] 
for free-space propagation and has been extended to multipath channels in [3]. The difference between those works and our is that they rely upon different assumptions. The main difference is the random assumption for the asynchronism, which they assume to be uniformly distributed over $\left[0, T_{f}\right)$, whereas we assume that it is uniformly distributed over $\left[0, N_{f} T_{f}\right)$. We think this last assumption is more appropriate since the code period is spread over duration $N_{f} T_{f}$, whereas [1] and [3] ones accounts for almost synchronized transmissions. They also assume that $N_{c} T_{c}<T_{f} / 2-2 T_{w}$ which corresponds to a guard time of more than $50 \%$ of the frame duration. This assumption along with the fact that the asynchronism is assumed to belong in $\left[0, T_{f}\right)$ allow to consider the frames as independent. Thus, although they consider the case $N_{f} \geq 1$, their approach corresponds to our case as if one frame occurs $\left(N_{f}=1\right)$.

\section{Optimal Time-HopPing CODES SELECTION}

Expression (15) clearly shows the contribution of the impulse shape through $\gamma_{w w}$, the codes through $\kappa_{1}$ and the channel through $\psi$. The interesting property of this expression is that the codes contribution appears in factor of the other terms and thus can be optimized independently from the channel and the pulse shape. We can thus identify a criterion that we may enable us to select pairs of codes, $\left(\mathbf{c}_{1}, \mathbf{c}_{n}\right)$, which minimizes $\kappa_{1}$, where $\mathbf{c}_{n}=\left[c_{n}(0), \cdots, c_{n}\left(N_{c} N_{f}-1\right)\right]$ denotes the DTHC vector of user $n$. This criterion is equivalent to minimizing for all $n, \kappa_{1, n}$. We have demonstrated that the minimum value of $\kappa_{1, n}$ is equal to $N_{f}^{2}$ (see [5] for details), thus we can deduce of it, that the minimum MUI variance is achieved if and only if the set of pair of codes $\left\{\left(\mathbf{c}_{1}, \mathbf{c}_{n}\right), n=2, \cdots, N_{u}\right\}$ verifies the condition, $\kappa_{1, n}=N_{f}^{2}$. A pair of codes verifying $\kappa_{1, n}=N_{f}^{2}$ is called an "optimal pair".

Notice that, we have established a criterion that enables us to find optimal pair, but it does not provide any method for constructing the optimal codes. However, for a given user $n_{0}$ it is possible, via exhaustive search to find the set of all the optimal pairs $\left(\mathbf{c}_{n_{0}}, \mathbf{c}_{m}\right)$ for reasonnable values of $N_{c}$ and $N_{f}$. As soon as $N_{c}$ and/or $N_{f}$ is too large, exhaustive search fails and elaborating a low-complex constructive method is still an open issue.

All the users (or nodes) belonging to this set, constitutes a network where the users can transmit at the same time while user $n_{0}$ is able to demodulate any subset, while experimenting the least MUI possible.

In Fig. 1, we display the percentage of all possible optimal pairs versus $N_{c}$ for $N_{f}=3$ and $N_{f}=4$. We observe that this percentage increases when $N_{c}$ increases. Consequently, as soon as $N_{c}$ is large enough, the optimal pairs are not seldom.

As an example, for $N_{f}=3$ and $N_{c}=18$, we have found a set of optimal pairs of codes with $N_{u}=7$ users. The set is as follows: $\tilde{c}_{1}=\{0,0,2\}, \tilde{c}_{2}=\{0,1,4\}, \tilde{c}_{3}=\{0,4,1\}$, $\tilde{c}_{4}=\{0,5,10\}, \tilde{c}_{5}=\{0,6,12\}, \tilde{c}_{6}=\{0,7,14\}$, and $\tilde{c}_{7}=$ $\{0,8,16\}$.

We can also remark that in earlier works, due to the assumptions the collision term $\kappa_{1}$ is always equal to 1 , and thus all the pairs are optimal. Therefore their expression of the MUI variance does not depend upon the codes [1], [3]. As a conclusion, our result (15) is novel since it is adapted to UWB Impulse Radio scheme, and is useful since it enables us to distinguish the "good" codes from the "bad" codes in a realistic propagation scheme.

\section{Simulations}

In this section, we analyze through simulations the influence of the codes on the error probability.

Like [8], we compute the true error probability at the output of the rake receiver. It is given by $P_{e}(a, \tau, d, \theta, n)=\operatorname{Pr}\{z<$ $0 \mid a, \tau, d, \theta, n\}$, assuming that $d_{1}(0)$, the transmitted symbol of the user of interest, is fixed and is equal to $d_{1}(0)=1$. The average error probability denoted $\bar{P}_{e}$ is then obtained by averaging $P_{e}(a, \tau, d, \theta, n)$ over all the random variables and can be written $\bar{P}_{e}=\mathbb{E}_{a, \tau, d^{*}, \theta^{*}, n}[\operatorname{Pr}\{z<0 \mid a, \tau, d, \theta, n\}]$ where $\mathbb{E}[$.$] stands for the empirical mean and where d^{*}=$ $d \backslash\left\{d_{1}(0)\right\}$ and $\theta^{*}=\theta \backslash\left\{\theta_{1}\right\}$. As the noise $n$ is zero-mean Gaussian, $\bar{P}_{e}$ reduces as follows:

$$
\bar{P}_{e}=\mathbb{E}_{a, \tau, d^{*}, \theta^{*}}\left[\frac{1}{2} \operatorname{erfc}\left(\frac{z_{1}+z_{2}+z_{3}}{\sqrt{2} \sigma_{\eta}}\right)\right],
$$

where $\sigma_{\eta}^{2}$ is the variance of the filtered Gaussian noise $\eta$.

By considering that optimizing the variance of the MUI with respect to the THC is a relevant task, we have implicitly assumed that the encountered MUI is Gaussian (as in [4] and [3]). Unfortunately as observed in [9], the MUI is not Gaussian and the true error probability given by (19) is actually much larger than the error probability calculated under the Gaussian MUI assumption. According to simulation results, we will see that the codes optimization based on the minimization of the MUI variance remains valid in practice, i.e., the error probability (19) for optimized codes is much smaller than for the non-optimized ones.

For the simulations, we consider the channel model CM2 proposed in [6] with only one cluster. We consider a selective rake (SRAKE) receiver with $L_{r}=3$ fingers. Parameters of the Gaussian pulse $w(t)$ are chosen such that the pulse spectrum fits the shape of the FCC spectral mask [10]. For practical purpose, the pulse (with unitary energy) is truncated to duration $T_{w}=1 \mathrm{~ns}$, and thus, can be written as:

$w(t)=\frac{2 \sqrt{2}}{\sigma^{2} \pi} \cos \left(2 \pi f_{0}\left(t-T_{w} / 2\right)\right) e^{-\left(t-T_{w} / 2\right)^{2} / 2 \sigma^{2}} \times \mathbb{1}_{\left[0, T_{w}\right]}$,

with $\sigma^{2}=911 \times 10^{-4}$ ns and $f_{0}=6.85 \mathrm{GHz}$.

The frame duration is $T_{f}=60 \mathrm{~ns}$. We put $N_{f}=3, N_{c}=18$ and $N_{u}=7$. In the sequel, we consider only one user of interest, namely, the user 1 . Consequently the number of pairs of codes is equal to 6 .

To check the benefit of our code optimization procedure, we have inspected the error probability of the user 1 for three different configurations: in the first configuration (denoted 0/6), all the interfering users use non-optimal pairs of codes; in the second configuration (denoted $3 / 6$ ), half of the interfering users use optimal pairs of codes; and in the third configuration 


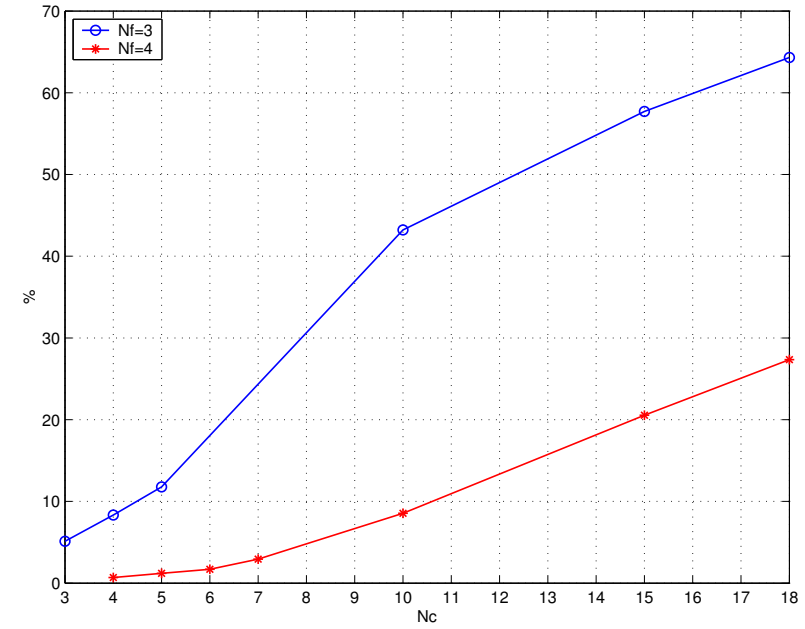

Fig. 1. Percentage of optimal pairs of codes vs $N_{c}$

(denoted 6/6), all the interfering users use optimal codes. Obviously this last configuration is the best one.

In Fig. 2, the average error probability $\bar{P}_{e}$ is plotted versus the average received bit energy to noise ratio, $\bar{E}_{b} / N_{0}$ with $\bar{E}_{b}=\mathbb{E}_{a, \tau, d, \theta}\left[\int_{0}^{N_{f} T_{f}} r_{1}^{2}(t) d t\right]$ where $r_{1}(t)$ is the received signal associated with user 1 . For the channel model [6], we find $\bar{E}_{b}=N_{f} r_{w w}(0) \cdot \sum_{k=1}^{N_{p}} \mathbb{E}_{\tau}\left[I_{1}^{k}\right]$ with $\mathbb{E}_{\tau}\left[I_{1}^{k}\right]=$ $\lambda^{k}(\lambda+1 / \gamma)^{-k}$. We remark that the performance improves as soon as some optimal pairs of codes are used.

\section{CONCLUSION}

A closed-form expression of the MUI variance at the output of a rake receiver has been derived for general assumptions on the channel and the rake receiver. The expression of the MUI variance shows that the code contribution appears in factor and is independent of the other parameters of the transmission scheme. We have deduced from this expression a practical criterion that enables us to select a set of optimal codes that ensures minimal MUI variance at the output of the rake receiver. Simulations have shown that using the codes which minimize the MUI variance leads to decrease the error probability significantly. Thus, the proposed criterion appears as a useful tool for designing THC in UWB-IR transmissions.

\section{REFERENCES}

[1] R. A. Scholtz, "Multiple access with time-hopping impulse radio," in Proc. of the Milcom Conf., Boston, MA, USA, pp. 447-450, Oct. 1993.

[2] C. J. Le Martret, and G. B. Giannakis, "All-Digital impulse radio for wireless cellular systems," IEEE Trans. on Communications, vol. 50, no. 9 , pp. 1440-1450, Sept. 2002.

[3] F. Ramírez-Mireles, "Error probability of ultra wideband SSMA in a dense multipath environment," in Proc. of the Milcom Conf. , vol. 2, Anaheim, CA, USA, pp. 1081-1084, Oct. 2002.

[4] M. Z. Win, and R. A. Scholtz, "Ultra-wide bandwith time-hopping spread-spectrum impulse radio for wireless multiple-access communications," IEEE Trans. on Communications, vol. 48, no. 4, pp. 679-691, April 2000.

[5] C. J. Le Martret, A.-L. Deleuze, and P. Ciblat, "Optimal time-hopping code design for multi-user interference mitigation in Ultra-Wide Band Impulse Radio", submitted for publication to IEEE Trans. on Wireless Communications, June 2004.

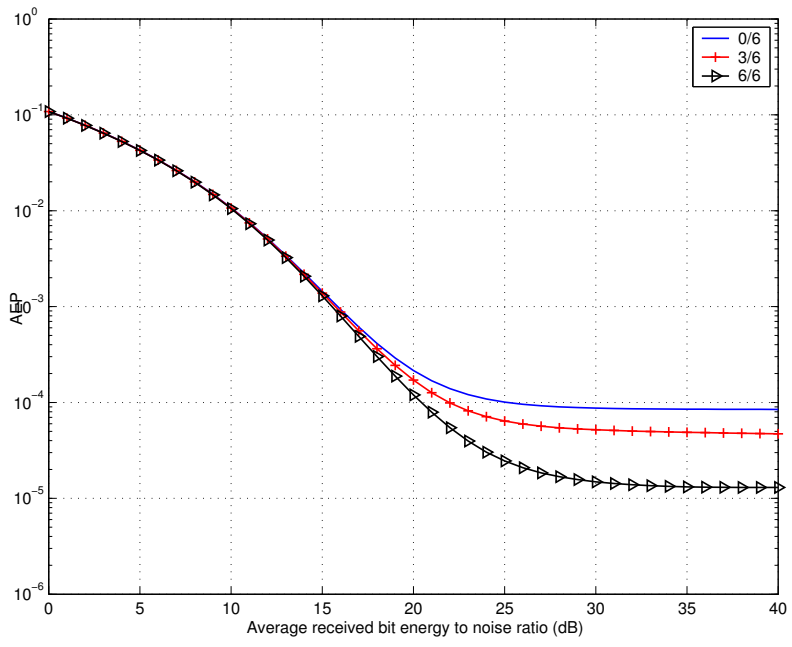

Fig. 2. Average Error probability vs. $\bar{E}_{b} / N_{0}$ for SRAKE receiver with $L_{r}=3$ fingers, $N_{u}=7, N_{f}=3$ and $N_{c}=18$.

[6] A. F. Molish, J. R. Foerster, and M. Pendergrass, "Channel models for ultrawideband personal area networks," IEEE Wireless Communications, vol. 10, Dec. 2003.

[7] A. A. M. Saleh, and R.A. Valenzuela, "A statistical model for indoor multipath propagation," IEEE Journal on Selected Areas in Communications, vol. SAC-5, no. 2 pp. 128-137, Feb. 1987.

[8] G. Durisi, and S. Benedetto, "Performance evaluation of TH-PPM UWB systems in the presence of multiuser interference," IEEE Communications Letters, vol. 7, no. 5, pp. 224-226, May 2003.

[9] G. Durisi, and G. Romano, "On the validity of Gaussian approximation to characterize the multiuser capacity of UWB TH-PPM," in Proc. IEEE Conference on Ultra Wideband Systems and Technologies 2002, Digest of Papers, Baltimore, USA, pp. 157-162, May 2002.

[10] Federal Communication Commission, "Revision of Part 15 of the commission's rules regarding ultra-wideband transmission systems, first Report and Order," FCC, Feb. 2002. 